\title{
X-Ray Emission Spectrometer Design with Single-Shot Pump-Probe and Resonant Excitation Capabilities
}

\author{
Katherine Spoth \\ Office of Science, Science Undergraduate Laboratory Internship (SULI) \\ State University of New York at Buffalo \\ SLAC National Accelerator Laboratory \\ Menlo Park, CA
}

August 12, 2011

Prepared in partial fulfillment of the requirements of the Office of Science, Department of Energy's Science Undergraduate Laboratory Internship under the direction of Dennis Nordlund at the Stanford Synchrotron Radiation Lightsource, SLAC.

Participant:

Signature

Research Advisor:

Signature 


\section{TABLE OF CONTENTS}

Abstract

$\begin{array}{ll}\text { Introduction } & 1\end{array}$

Materials and Methods 2

$\begin{array}{lc}\text { Results } & 5\end{array}$

$\begin{array}{ll}\text { Discussion and Conclusions } & 10\end{array}$

$\begin{array}{ll}\text { Acknowledgments } & 11\end{array}$

$\begin{array}{ll}\text { References } & 11\end{array}$ 


\begin{abstract}
X-Ray Emission Spectrometer Design with Single-Shot Pump-Probe and Resonant Excitation Capabilities. KATHERINE SPOTH (State University of New York at Buffalo, Buffalo, NY 14260) DENNIS NORDLUND (Stanford Synchrotron Radiation Lightsource, SLAC, Menlo Park, CA 94025)
\end{abstract}

Core-level spectroscopy in the soft X-ray regime is a powerful tool for the study of chemical bonding processes. The ultrafast, ultrabright X-ray pulses generated by the Linac Coherent Light Source (LCLS) allow these reactions to be studied in greater detail than ever before. In this study, we investigated a conceptual design of a spectrometer for the LCLS with imaging in the non-dispersive direction. This would allow single-shot collection of X-ray emission spectroscopy (XES) measurements with varying laser pump X-ray probe delay or a variation of incoming X-ray energy over the illuminated area of the sample. Ray-tracing simulations were used to demonstrate how the components of the spectrometer affect its performance, allowing a determination of the optimal final design. These simulations showed that the spectrometer's non-dispersive focusing is extremely sensitive to the size of the sample footprint; the spectrometer is not able to image a footprint width larger than one millimeter with the required resolution. This is compatible with a single shot scheme that maps out the laser pump X-ray probe delay in the non-dispersive direction as well as resonant XES applications at normal incidence. However, the current capabilities of the Soft X-Ray (SXR) beamline at the LCLS do not produce the required energy range in a small enough sample footprint, hindering the single shot resonant XES application at SXR for chemical dynamics studies at surfaces. If an upgraded or future beamline at LCLS is developed with lower monochromator energy dispersion the width can be made small enough at the required energy range to be imaged by this spectrometer design. 


\section{INTRODUCTION}

Understanding the bonding processes that take place during the formation of chemical compounds can give us a greater ability to predict their chemical properties and reactivity. Some knowledge of these processes can be gained by studying changes in molecular orbitals. Many of these interesting reactions involve $\mathrm{C}, \mathrm{N}$, and $\mathrm{O}$ atoms. Core level soft X-ray spectroscopy techniques allow us to study these systems in an atom-specific way, meaning we can probe a specific energy transition by creating a core hole that can only be filled by a nearby valence electron [1]. The transfer of electrons during bonding processes occurs on a femtosecond time scale. Measurements on this scale are made possible using ultrafast X-ray pulses generated by the Linac Coherent Light Source (LCLS). A spectrometer that allows for Resonant Inelastic X-Ray Scattering (RIXS) or time-resolved X-Ray Emission Spectroscopy (XES) measurements to be taken in a single shot at the LCLS will allow us to study changes in the electronic structure of complex systems in greater detail than has ever been possible.

One of the many systems that would be interesting to study using this instrument is the dissociation of $\mathrm{N}_{2}$ as occurs during the Haber-Bosch process through which ammonia is produced from nitrogen and hydrogen on a substrate of ruthenium or iron [2]. This process is widely used in the production of nitrogen-based fertilizers responsible for growing crops that feed much of the world's population and therefore is estimated to account for as much as one percent of the world's total energy consumption [3]. For this reason, it would be very beneficial if we could make improve this process to reduce its energy consumption.

The processes that take place during this and other surface reactions are difficult to observe experimentally. Between adsorption and desorption steps, charge and energy transfer takes place between the adsorbates and catalyst determining the intermediate steps in the reaction. These short-lived elementary steps can be observed using the time-resolved measurements made possible in ultrafast laser pump X-ray probe experiments using XES at the 


\section{LCLS.}

Our goal is to determine the best design of a new XES spectrometer for the LCLS. We modify the design of a typical XES spectrometer to allow for single-shot measurements with a time delay or energy gradient along the sample footprint. Ray-tracing simulations of possible configurations are used to determine the best design, which has maximum throughput at the energy and imaging resolutions required to perform the desired experiments.

\section{MATERIALS AND METHODS}

Figure 1 shows an illustration of the X-ray emission process. In non-resonant X-ray emission, incoming X-ray photons excite an electron from the core of an atom in a sample, ejecting the electron into the vacuum. In the resonant case, this electron is not ejected but promoted to an unoccupied atomic or molecular orbital [4]. This is achieved by using a resonant incoming $\mathrm{X}$-ray energy, which is equal to the difference between the core level energy and that of the excited state. In both cases, an electron from the valence level falls to fill the vacancy in the core, radiating an X-ray photon with energy equal to the difference between its initial and final states. The XES spectrum shows the intensity vs. energy of these emitted X-rays. From this spectrum, the energy and symmetry of the atom's orbitals can be determined, providing information about its valence band electronic structure.

Because each element has a unique core level energy, XES is element-specific. When bonds are formed with another element, the energies of an atom's orbitals transform. These changes in energy can be easily observed using XES, making this method chemical bondingspecific as well. Additionally, because the core holes created in this method can only be filled by valence electrons in the vicinity of the atom, XES is a useful tool in probing the local electronic structure of an atom.

In a typical XES spectrometer, which is shown in Figure 2, incoming X-rays excite core 
electrons in the sample causing outer electrons to decay and emit X-rays. The emitted rays are incident on a concave grating, which disperses the photons according to energy and focuses the light in this dispersive direction. At the detector, the focused rays' positions are related to their energy due to the their interaction with the diffraction grating.

Figure 3 illustrates how the sample footprint can be used to image laser-X-ray time delay. The sample footprint is defined as the area which is illuminated by incoming X-rays. In regular pump-probe experiments, the sample is excited by a laser pulse then probed using $\mathrm{X}$-rays at a time delay. It is possible to create a varying time delay along the width $x$ of this sample footprint by placing the X-ray probe at an angle to the laser pump such that the pump arrives first, followed by the probe at a time delay that increases across the sample footprint via the speed of light. If the laser pump incidence is normal to the sample, the time delay can be described by the equation $\mathrm{TD}=\frac{x}{c}$. We can shorten this time delay by decreasing the incidence angle of the laser pump.

In a similar way we can also vary the energy of incoming X-rays along the sample footprint for RIXS measurements, shown in Figure 4. At the Soft X-Ray (SXR) beamline at LCLS, a monochromator tunes incoming X-ray energy by dispersing the photons with a grating and selecting the desired energy using an entrance slit. By increasing the size of this slit, a range of energies ( $5 \mathrm{eV}$, for example) can be used to illuminate the sample with incoming energy related to position $x$ on the footprint. The footprint width is dictated by the energy dispersion of the monochromator and the angle of the sample to the incoming beam. To achieve an energy difference of $2 \mathrm{eV}$ at the SXR beamline a footprint with width of about 2 mm must be used.

The conceptual design of the proposed spectrometer is shown in Figure 5. Imaging over the sample footprint is in the non-dispersive direction of the grating; light in the dispersive direction, which corresponds to vertical on the sample, is dispersed by the grating according to its energy and focused. In the typical spectrometer design, rays are not focused in the 
non-dispersive direction. In order to have a meaningful picture on the detector that gives some information about the sample at increasing time delay or energy, the light in this non-dispersive direction must be focused. This is achieved by adding a concave mirror perpendicular to the grating. The result is an image on the detector in which many XES spectra can be observed as a function of incoming energy or pump-probe delay.

Due to the low cross-section of the X-ray emission technique, one of the most important aspects in the design of the spectrometer is to maximize throughput while maintaining an energy resolution small enough to study the systems of interest. Figure 5 includes a summary of the desired resolution. In the case of surface reactions involving adsorbates on catalysts studied here, $0.25 \mathrm{eV}$ is the best resolution required. It is also useful in some cases to allow high throughput measurements at up to $1 \mathrm{eV}$ resolution. For time-resolved experiments, we define a resolution of $10 \mu \mathrm{m}$ on the source in the non-dispersive direction corresponding to a 30 fs time-delay (Figure 3). For the RIXS case with varying energy along the sample footprint we wish to have a $0.25 \mathrm{eV}$ incoming energy resolution. Using the SXR beamline design and an incidence angle of $12^{\circ}$, we define this as $200 \mu \mathrm{m}$. For the sake of clarity, we refer to resolution in the dispersive direction as the energy resolution of the spectrometer and to resolution in the non-dispersive direction as imaging resolution, which for the RIXS case is in units of energy. Additionally, the spectrometer will be optimized for use at 520 $\mathrm{eV}$, the oxygen K-line.

To analyze the possible designs of the spectrometer, we performed ray-tracing simulations using the SHADOWVUI extension of X-Ray Oriented Programs (XOP) [5]. We tested several illumination distances for the grating and for the non-dispersive focusing mirror. We also looked at the effect of changing the mirror's position, causing changes in the magnification at the detector, and investigated several possible mirror shapes. 


\section{RESULTS}

The final design of the spectrometer has three main components: the imaging mirror, dispersive grating, and detector. The detector to be used has already been specified-a $4 \times 4 \mathrm{~cm}$ multichannel plate used with a CCD camera to produce a picture of intensity vs. position in two dimensions. Therefore, our main focus has been analyzing the performance of the dispersive grating and the imaging mirror.

\section{Dispersive Grating}

The most basic spectrometer design consists of a dispersive grating and detector; this is a useful starting point for the new design. We choose three parameters of the grating that will dictate the geometry of the entire spectrometer: the radius of curvature $R$ at the center, the ruling spacing $d$, and the incidence angle $\alpha$. From these parameters one can mathematically determine the total length of the spectrometer and the energy dispersion at the detector. The energy dispersion describes the way that the vertical detector position changes with photon energy. For soft X-ray applications the incoming angle must be small because many of the X-rays will be absorbed by the grating material. This is limited by using grazing incidence near or below the material's critical angle. For a nickel coated mirror at $520 \mathrm{eV}$ this angle is on the order of $4^{\circ}$; above this angle the reflectivity decreases sharply. This design uses an incidence angle of $5^{\circ}$, or $\alpha=85^{\circ}$ ( $\alpha$ is defined relative to normal incidence).

We define the radius of curvature $R$ and the ruling spacing $d$ based on the spectrometer's desired length and dispersion. We wish to have a total length close to 1 meter and an energy dispersion of about $0.4 \mu \mathrm{m} / \mathrm{meV}$. A reasonably small source size (about $10 \mu \mathrm{m}$ ) should have an energy resolution close to $0.25 \mathrm{eV}$, the high-resolution design goal. This fact dictates the energy dispersion required of the grating. The proper spectrometer length and energy dispersion are achieved with $R=7.5 \mathrm{~m}$ and a grating spacing of 800 rulings per mm. 
From $\alpha, R$, and $d$ we can determine the correct position of the sample, grating, and detector such that the light coming off of the grating is focused at the detector. These positions are determined using the Rowland Circle geometry shown in Figure 6. For a grating with radius of curvature $R$ there exists a circle of diameter $R$ such that if the source of light is on this circle, then the diffracted image in all orders will be in focus at points on the circle. From these conditions, we can calculate the correct positions of the grating and detector relative to the sample (summarized in Figure 6). The spectrometer is operated in negative order to increase the distance between the sample and grating, which is necessary for the inclusion of the non-dispersive focusing mirror.

The grating's shape is that of a symmetric ellipse. This shape produces lower spherical aberrations than a spherical reflector would, allowing a longer section of the grating to be illuminated before similar errors to a sphere are produced. A longer illumination distance allows more rays to strike the grating, resulting in a higher overall throughput. The best shape of this ellipse is determined through calculations that show the energy dispersion at different offsets from the center of the grating. The relationship between the major and minor axes' lengths is constrained by the requirement that the curvature at the center of the grating be equal to $7.5 \mathrm{~m}$. This allows us to manipulate the length of the major axis of the ellipse and see the effect on the energy dispersion. The best shape is that for which the dispersion is symmetric about the center of the grating. In this case, we use a major axis of about $55 \mathrm{~cm}$ for which the corresponding minor axis length is $4 \mathrm{~cm}$.

\section{Imaging Mirror}

To allow for imaging in the non-dispersive direction, we consider a mirror reflecting this direction, focusing at the same spot as that defined by the grating. The position, shape, and grazing are chosen such that light from the source will be focused at the position of the detector. The mirror's position also determines the magnification at the detector. 
The "perfect" shape of the mirror is dictated by the source distance, the detector (image) distance, and the incidence angle. For the same reasons as in the case of the grating, a very grazing incidence angle must be used; here we choose $4^{\circ}$ again based on the reflectivity of a nickel coated mirror. SHADOW calculates the ideal shape of the mirror given its geometry (elliptical or spherical), the object and image focus distances, and the incidence angle. We analyzed the performance of three possible mirror shapes: a sphere, a symmetric ellipse, and an ideal ellipse. Figure 7 illustrates the distinction between the ideal and symmetric ellipses. What we call an ideal ellipse is the small section of the ellipse with foci at the sample and the detector for which light from the sample is incident at the specified angle. The symmetric ellipse is a section of the ellipse centered at one of the ellipse's axes, thus its curvature is symmetric about its center.

The first case simulated in SHADOW was 1:10 imaging for an ideal elliptical mirror at $4^{\circ}$ incidence. In addition to viewing the image spot at the detector we used a SHADOW post processor called FOCNEW to determine if the mirror was focusing as required. FOCNEW computes the waist size of the X-ray beam as a function of its position from an optical element and returns the position in which this size is smallest. Based on the parameters of the mirror, the best focus should be at the same location as the detector.

The result of analysis in FOCNEW was unexpected: the mirror's focusing ability was extremely sensitive to source's size and angular divergence. Specifically, if a source divergence of $0.015 \mathrm{rad}$ is used the position of best focus deviates from the detector position for any source size larger than $10 \mu \mathrm{m}$. This sensitivity is related to the departure of an ideal point source towards a source that is closer to a plane wave. This issue is limited to the width of the source as this is the direction in which this mirror is focusing; changing the source height or divergence in the vertical direction has no effect on the focus. Further simulations in SHADOW showed that for larger source sizes up to $1 \mathrm{~mm}$ the use of a larger divergence (around $0.15 \mathrm{rad}$ ) corrected the focusing issue, however here the detector image was very 
distorted and asymmetrical.

To better understand how the imaging distortion affects the performance of the spectrometer we carried out a series of simulations in which for different magnifications we determined the maximum divergence that could resolve $200 \mu \mathrm{m}$ on the source, conforming to the design goal. For example, in the 1:10 case, a spot $200 \mu \mathrm{m}$ on the source corresponds to a $2 \mathrm{~mm}$ spot on the detector, thus we determined which source divergence produced a $2 \mathrm{~mm}$ spot. For these simulations, we used a point source $500 \mu \mathrm{m}$ offset from the center, i.e. the edge of a $1 \mathrm{~mm}$ sample footprint. Our goal was to determine if a smaller magnification would decrease the effects of this focusing problem. The results can be seen in Table 1. The maximum divergence remained steady around 0.01 rad for magnifications between 1:10 and 1:2 but decreased in the demagnification cases. Based on these results there is no reason to use a smaller magnification than 10, since it would decrease the achievable resolution without improving the instrument's acceptance. A magnification higher than 10 is impractical, as the mirror would need to be placed too near the sample.

We also investigated the effect of the mirror's shape on focusing, with the goal of determining whether a spherical or symmetric elliptical mirror would perform better than the ideal ellipse. For the spherical mirror, SHADOW calculates the proper radius based again on the image and object focal distances and the incoming angle. The proper shape of the symmetric elliptical mirror can be determined using the radius that SHADOW calculates for the spherical mirror as the radius of curvature of the center of the ellipse. From this we know the Rowland circle radius and can find the major and minor axis lengths. In the 1:10 case using a $5 \mu \mathrm{m}$ source, neither the spherical nor symmetric elliptical mirror was able to produce a source resolution of $200 \mu \mathrm{m}$ for any divergence greater than $0.001 \mathrm{rad}$. Based on this observation, alternative shapes to the ideal ellipse were discarded.

From these results we can conclude that of the configurations that were investigated, the 1:10 ideal ellipse at $4^{\circ}$ is the best choice for the non-dispersive focusing in this design. 


\section{Resolution Determination}

The proper length of both the mirror and the grating now must be determined. Here, the balance between resolution and flux has a large impact-longer illumination distances on the optics will create high throughput but also cause imaging error through spherical aberrations present when concave optics are illuminated far from their centers. We optimized this balance by finding the longest illumination of the mirror and grating conforming to the previously listed energy and imaging resolutions required for the different applications (Figure 5). This is achieved by simulating the resolution at different illumination lengths on both the mirror and the grating. The results are shown in Table 2. We examine the case of a point source, a small source (50 $\mu \mathrm{m}$ offset point source) as in the time-resolved case, and a large source (RIXS case, $500 \mu \mathrm{m}$ offset). The resolution is measured by finding the full width at half maximum (FWHM) of the spot on the detector in each axis (vertical corresponds to imaging, and horizontal to energy). These lengths can then be converted to time or energy resolutions using the relationships between sample position and energy in the non-dispersive direction, or the calculated energy dispersion in the dispersive direction. Usable combinations of illumination lengths are those for which the resolution is lower then the specified requirements.

According to this data, it appears as though the grating can be illuminated up to 16 $\mathrm{cm}$ and still have the appropriate resolution. However, at illuminations above $10 \mathrm{~cm}$ an asymmetry appears in the detector image due to spherical aberrations. This asymmetry is not easily identifiable in the numerical data because the FWHM was used to find the spot size, and the intensity of the asymmetry is low enough that it does not significantly alter the value of the FWHM. Figure 8 shows the detector footprint for a $10 \mathrm{~cm}$ grating illumination and for a $12 \mathrm{~cm}$ illumination. Here, it is easy to see the asymmetry that appears. One can determine whether it is beneficial to illuminate more than $10 \mathrm{~cm}$ of the grating by looking only at the contribution of the outer regions. If the main contribution of these regions is to 
the asymmetry in the image rather than to the peak then it is not useful to include them. The results of a simulation in which only the outer $1 \mathrm{~cm}$ of the grating was illuminated are shown in the graph in Figure 9. Around an illumination of $12 \mathrm{~cm}$, once can see that the position of the image begins to deviate significantly from the center. Therefore, it is wise to exclude any length greater than $12 \mathrm{~cm}$ on the grating for measurements requiring high energy resolution. For applications in which a high resolution is less important, illuminating up to $18 \mathrm{~cm}$ of the grating allows greater throughput at resolutions up to $1 \mathrm{eV}$.

From these simulations, we determine that a grating illumination of $10 \mathrm{~cm}$ is acceptable for both of the spectrometer's applications. In the case of time-resolved XES, a time resolution around $30 \mathrm{fs}$ on the sample is achieved for a mirror illumination of $1.8 \mathrm{~cm}$. For the RIXS case, a resolution of $0.25 \mathrm{eV}$ is achievable with $3 \mathrm{~cm}$ illumination of the mirror.

\section{DISCUSSION AND CONCLUSIONS}

Figure 10 depicts the final schematic of the design, along with a summary of the parameters determined from ray-tracing simulations that optimize its performance. With these parameters, the instrument is able to image sample footprints smaller than $1 \mathrm{~mm}$. Under these conditions, time-resolved XES measurements can be performed as well as RIXS measurements in which a small spot size is possible. Figure 4 shows how this spot size is limited by the setup of the beamline at which these measurements are performed, providing a comparison between the SXR beamline of LCLS and beamline 13 at the Stanford Synchrotron Radiation Lightsource (SSRL). Currently, the energy spread per mm on the sample at SXR is such that to image an energy difference of $2 \mathrm{eV}$ a sample footprint of width $2 \mathrm{~mm}$ or more would be required. This width can be minimized by placing the sample at normal incidence to the X-ray beam, however this would prevent the study of surface catalysis for which grazing incidence on the sample is needed. The setup at SSRL beamline 13 allows an 
acceptably small footprint size for these measurements, which allows steady state RIXS as well as picosecond time resolved measurements using the low-alpha operational mode of the Stanford Positron-Electron Accelerating Ring (SPEAR). However, LCLS's peak power and femtosecond resolution are ultimately needed for many of the prospective chemical dynamics studies considered here. At the SXR beamline, applications to surface reactions would be possible with an upgraded monochromator having lower energy dispersion (i.e. more eV per

$\mathrm{mm}$ ). A beamline at the future LCLS-II could also be designed with dispersion low enough to allow these measurements. The realization of one of these possibilities will make this spectrometer a powerful tool for the study of chemical systems.

\section{ACKNOWLEDGMENTS}

This research was completed at SLAC National Accelerator Laboratory between June and August 2011. Many thanks to my mentor, Dennis Nordlund, for his enthusiasm and guidance. I'd also like to acknowledge the funding provided to the SULI program by the U.S. Department of Energy, and thank the staff at SLAC who make this program such a success. Finally, thanks to my fellow SULI students for making this summer so memorable.

\section{REFERENCES}

[1] A. Nilsson, "Applications of core level spectroscopy to adsorbates," Journal of Electron Spectroscopy and Related Phenomena, vol. 126, no. 1-3, pp. 3 -42, 2002. [Online]. Available: http://www.sciencedirect.com/science/article/pii/S036820480200141X

[2] S. Dahl, A. Logadottir, R. C. Egeberg, J. H. Larsen, I. Chorkendorff, E. Törnqvist, and J. K. Nørskov, "Role of steps in N2 activation on Ru(0001)," Phys. Rev. Lett., vol. 83, no. 9, pp. 1814-1817, Aug 1999. 
[3] R. Schrock, "Nitrogen fix," Technology Review, May 2006. [Online]. Available: http://www.technologyreview.com/article/16822/

[4] A. Nilsson and L. G. M. Pettersson, "Chemical bonding on surfaces probed by X-ray emission spectroscopy and density functional theory," Surface Science Reports, vol. 55, no. 2-5, pp. 49 - 167, 2004. [Online]. Available: http: //www.sciencedirect.com/science/article/pii/S0167572904000573

[5] M. Sanchez de Rio and R. J. Dejus, "Status of XOP: an X-ray optics software toolkit," in Proc. SPIE, vol. 5536, 2004, p. 171. [Online]. Available: http://dx.doi.org/10.1117/12.560903 


\section{FIGURES}
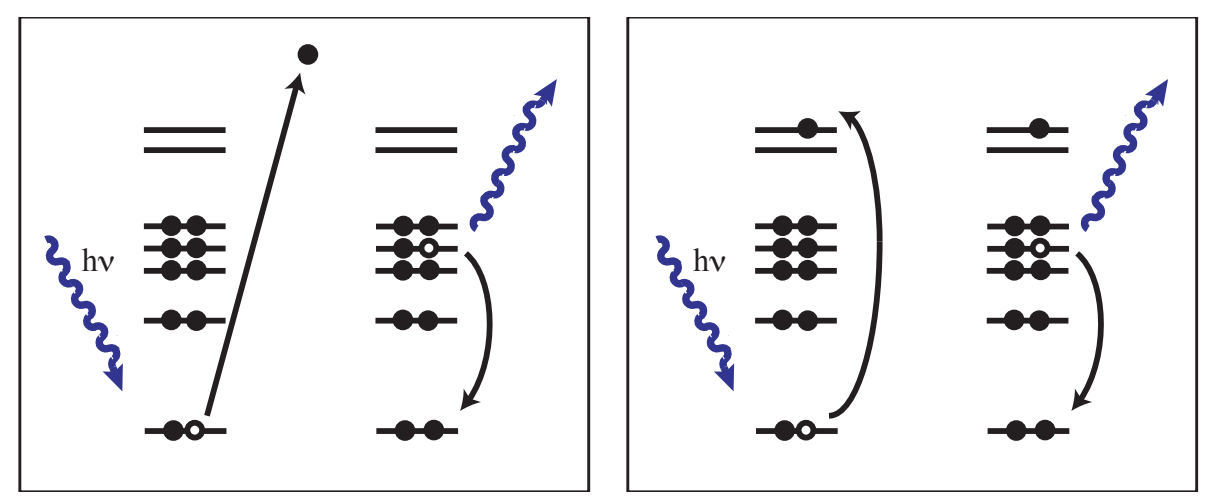

Figure 1: Illustration of the non-resonant (left) and resonant (right) cases of XES. Incoming X-ray radiation excites an electron from the atom's core level, ejecting it in the non-resonant case or promoting it to an unoccupied orbital level in the resonant case. A valence electron of the atom then falls to fill the core level vacancy, emitting a photon with energy equal to the energy difference between its initial and final states.

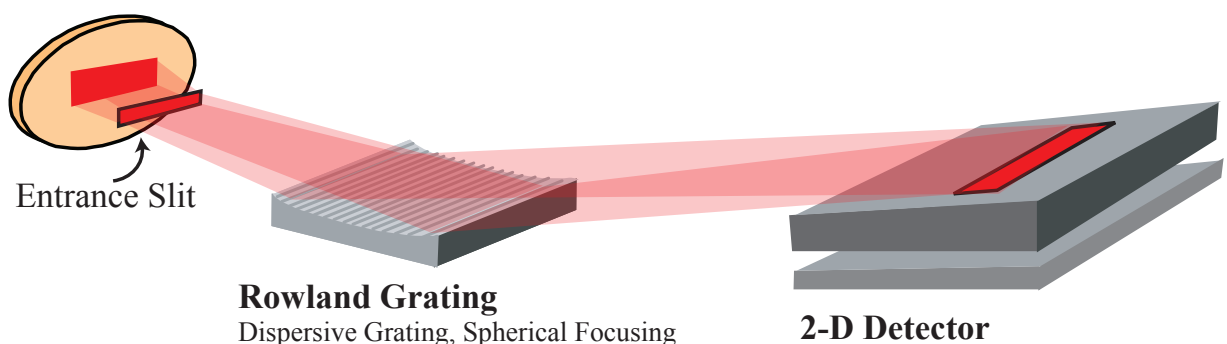

Figure 2: The basic schematic of a typical X-ray emission spectrometer, consisting of a dispersive grating and detector. 


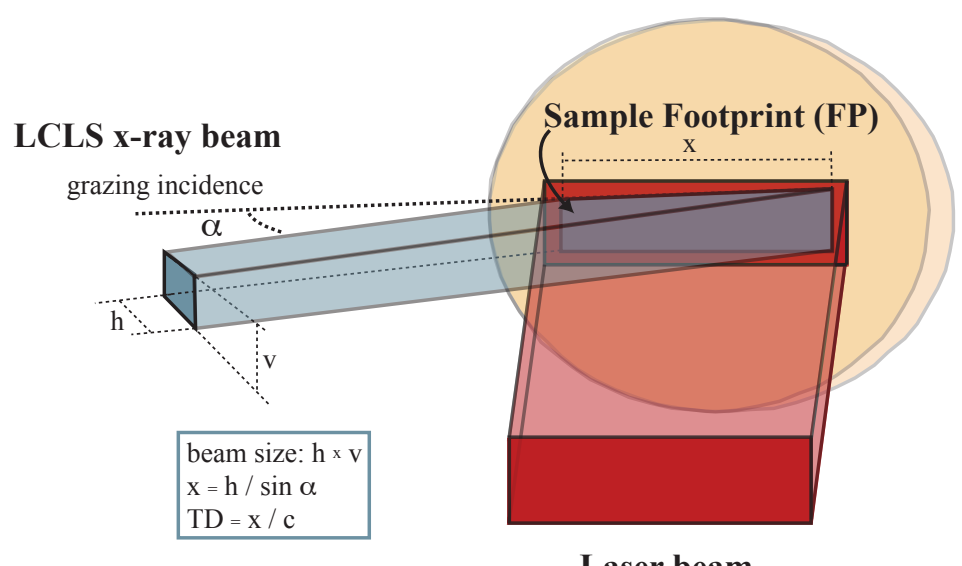

Laser beam

Figure 3: Illustration of imaging in the non-dispersive direction with variable time delay. For time-resolved imaging place the X-ray probe at an angle to the laser pump at normal incidence, causing the probe to arrive at a time delay linearly related to position $x$ on the footprint.

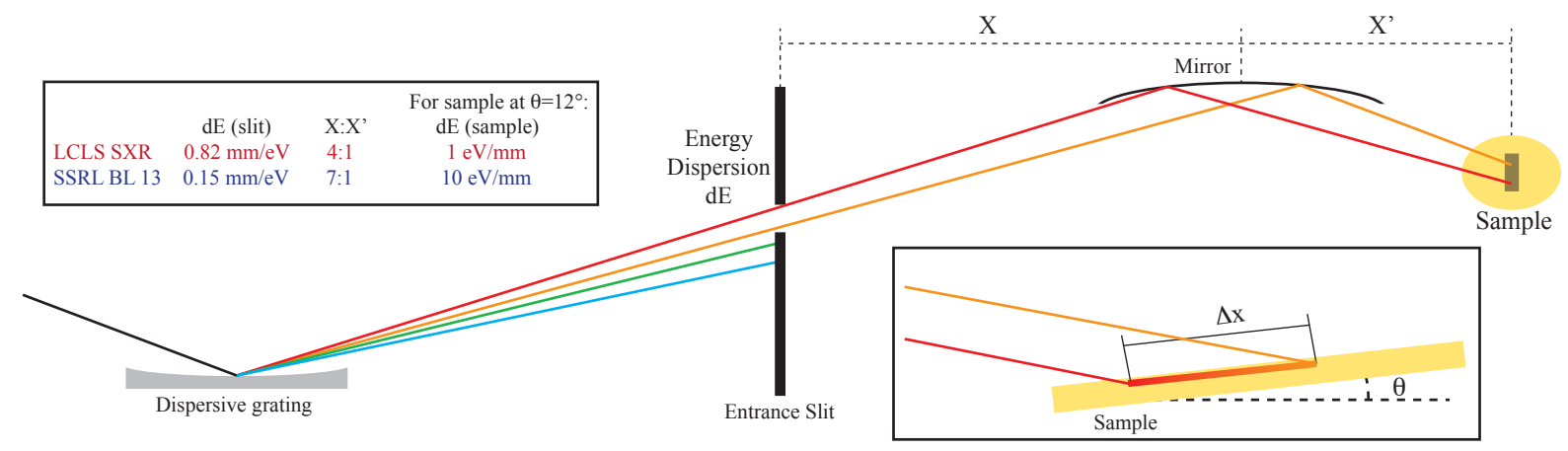

Figure 4: Illustration of the SXR beamline monochromator. Widening the entrance slit allows a range of wavelengths to illuminate the sample footprint. The footprint width is dictated by the monochromator's energy dispersion at the sample and the angle at which the sample is placed relative to the X-ray beam. The current setup at SXR allows $1 \mathrm{eV}$ to be imaged per $\mathrm{mm}$ on the sample; we also provide a comparison to the monochromator at SSRL beamline 13 for which the dispersion at the sample is ten times smaller. An improved setup would have the ability to produce a higher energy difference at more grazing incidence on the sample. 


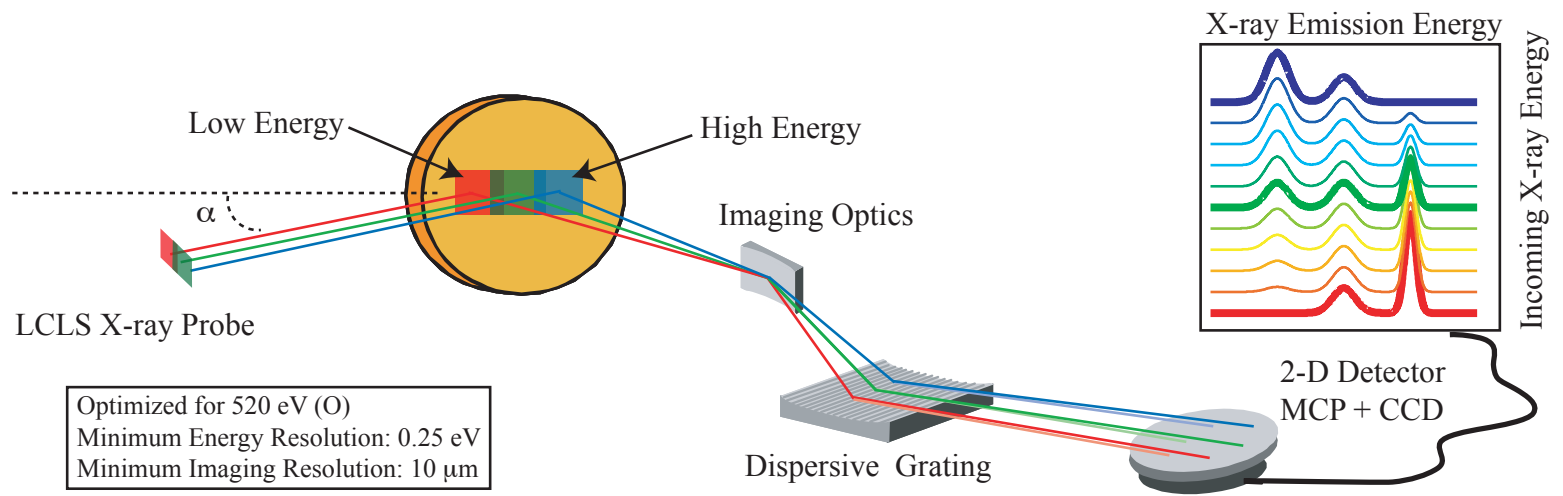

Figure 5: A schematic of the proposed spectrometer. The incoming X-rays vary in energy along the sample footprint $x$. X-rays emitted from the sample are focused in the nondispersive direction by a concave mirror. An elliptical grating disperses the rays according to energy and focuses them in this dispersive direction, producing the detector image shown in the figure. The time-resolved case is very similar: a laser pump is added at an angle to the X-ray probe such that time delay varies across $x$. The $2 \mathrm{D}$ detector displays X-ray probe delay in the vertical rather than incoming X-ray energy.

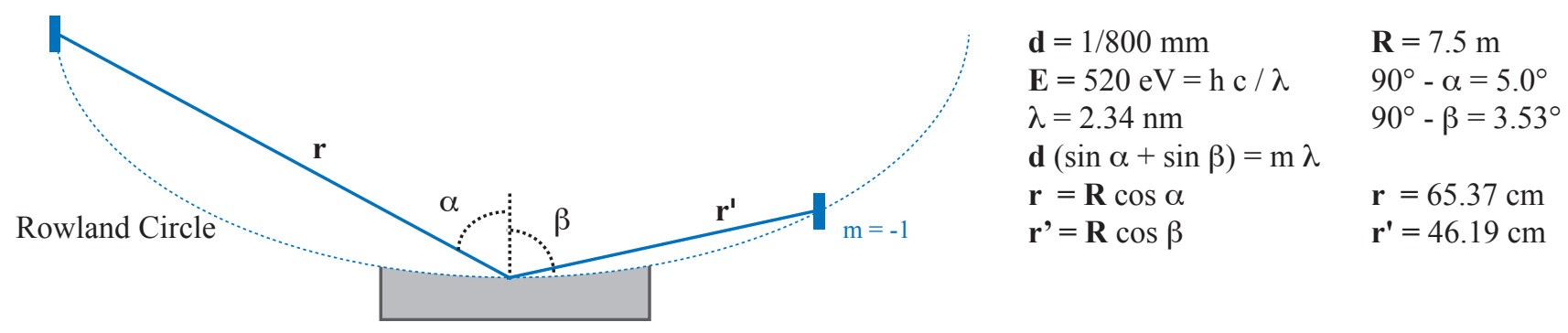

Figure 6: Illustration of the sample-grating-detector setup, showing the Rowland circle geometry and the details of calculations of the correct grating and detector positions. The Rowland Circle is a circle with radius one-half the radius of curvature of the center of the grating. If the source is located on this circle, the diffracted image in all orders will be in focus on the circle. 

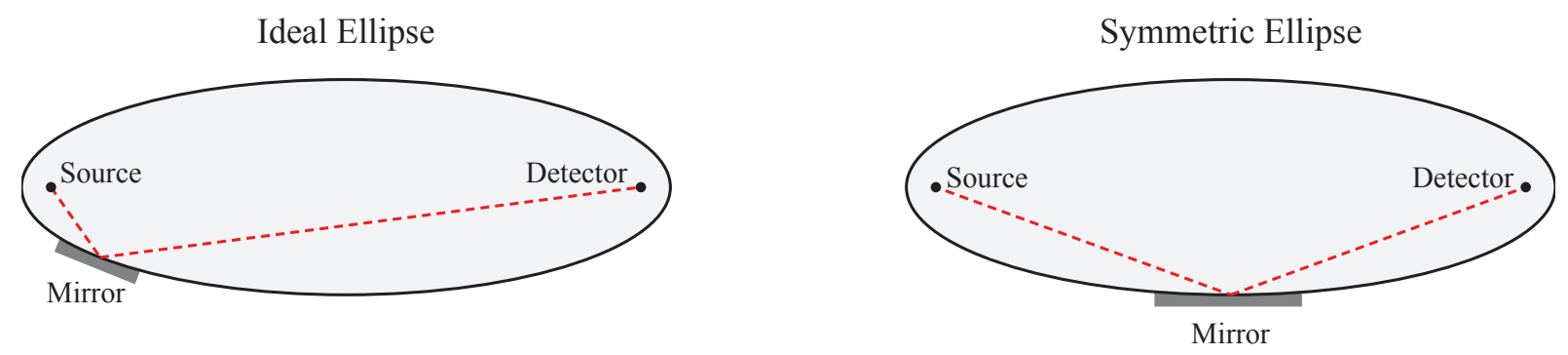

Figure 7: Distinction between symmetric and ideal elliptical geometries. In the proposed spectrometer design, the non-dispersive focusing mirror has an ideal elliptical shape, while the grating is a symmetric ellipse with focusing dictated by the Rowland condition
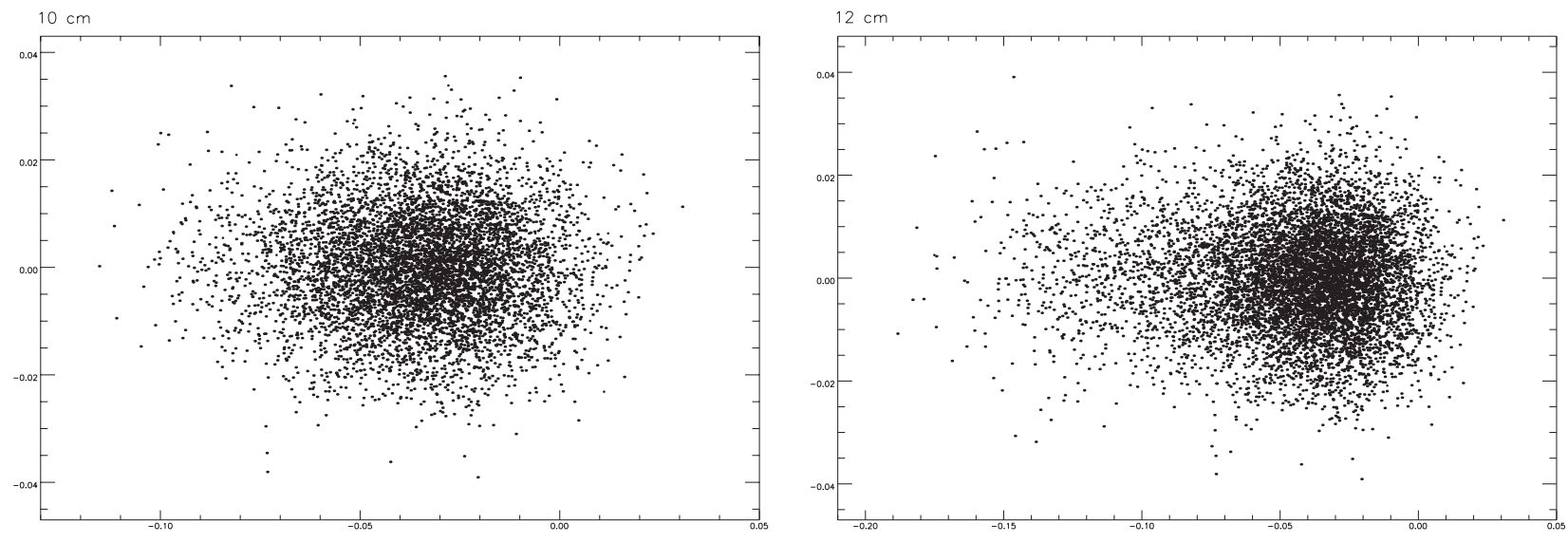

Figure 8: Detector footprints in SHADOW for a grating illumination of $10 \mathrm{~cm}$ (left) and 12 $\mathrm{cm}$ (right). These show the picture of the X-ray spot on the detector, with the dispersive direction being in the horizontal and the non-dispersive in the vertical. One can easily see the asymmetry that begins to appear in the $12 \mathrm{~cm}$ case due to aberrations resulting from the illumination of the grating far from its center. 


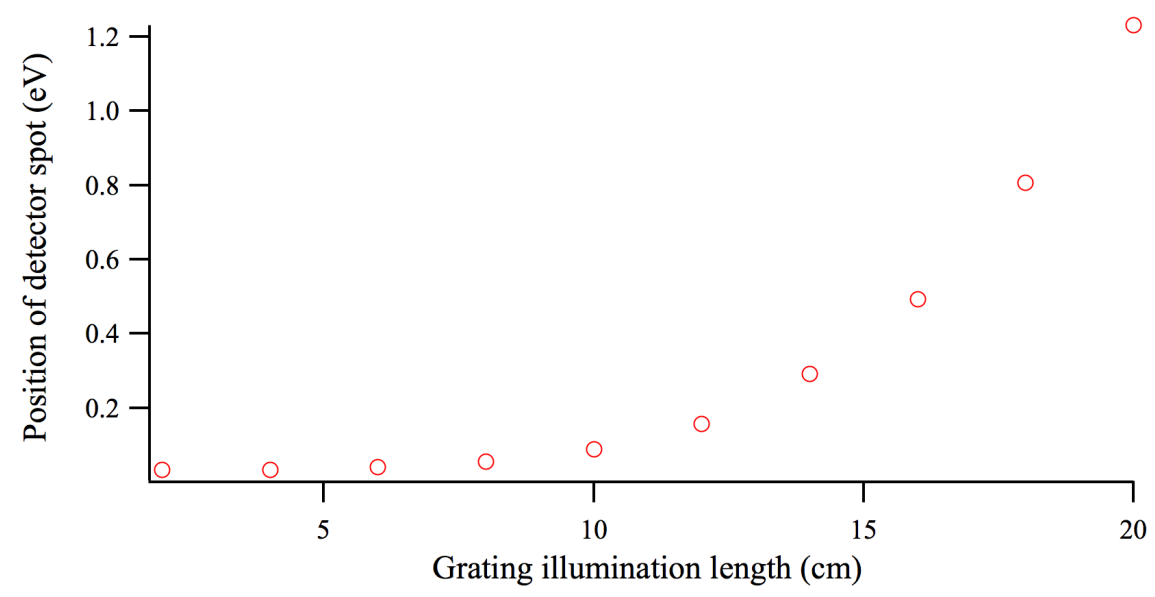

Figure 9: The contribution of only the outer $1 \mathrm{~cm}$ of the grating illumination to the detector spot size. If the position of the spot's center deviates significantly from its position when the center of the grating is illuminated, this section of the grating contributes mainly to the asymmetry in the image at the detector. The position is given in $\mathrm{eV}$ for ease of comparison to the design requirements. We see that for a resolution of $0.25 \mathrm{eV}$, we can illuminate $12 \mathrm{~cm}$ of the grating; for $1 \mathrm{eV}$ resolution up to $18 \mathrm{~cm}$ can be illuminated.

Top view:
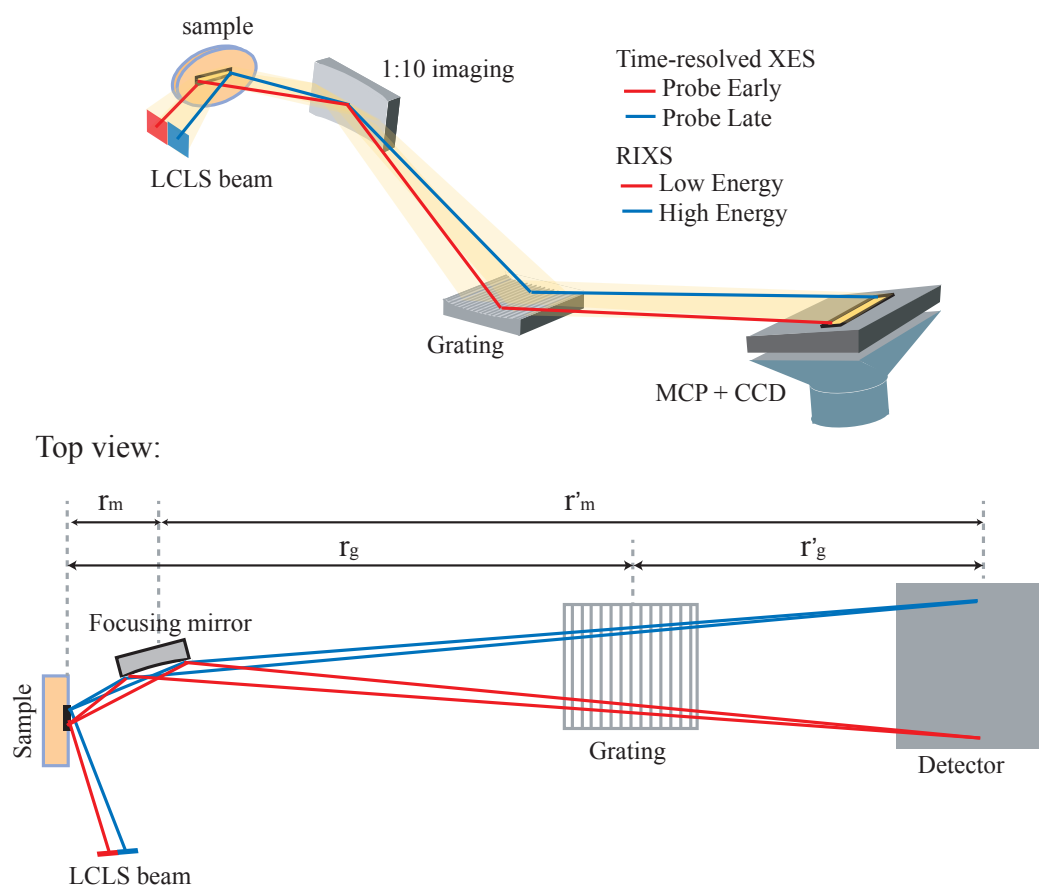

\begin{tabular}{|c|c|}
\hline \multicolumn{2}{|l|}{ Positions } \\
\hline $\mathrm{r}_{\mathrm{m}}:$ & $10.1 \mathrm{~cm}$ \\
\hline $\mathrm{r}_{\mathrm{m}}^{\prime \prime}:$ & $101.4 \mathrm{~cm}$ \\
\hline$r_{g}:$ & $65.4 \mathrm{~cm}$ \\
\hline $\mathrm{r}_{\mathrm{g}}^{\mathrm{g}}$ : & $46.2 \mathrm{~cm}$ \\
\hline \multicolumn{2}{|l|}{ Grating } \\
\hline Radius (R): & $7.5 \mathrm{~m}$ \\
\hline Major Axis: & $55.0 \mathrm{~cm}$ \\
\hline Minor Axis: & $4.0 \mathrm{~cm}$ \\
\hline Line density: & $800 \mathrm{~mm}^{-1}$ \\
\hline Incoming angle: & $5^{\circ}$ \\
\hline Outgoing angle: & $3.5^{\circ}(520 \mathrm{eV})$ \\
\hline \multicolumn{2}{|c|}{$\begin{array}{l}\text { Illumination distance: } 12 \mathrm{~cm} \\
\text { (high resolution) }\end{array}$} \\
\hline \multicolumn{2}{|c|}{$\begin{array}{l}\text { Illumination distance: } 18 \mathrm{~cm} \\
\text { (high throughput) }\end{array}$} \\
\hline \multicolumn{2}{|l|}{ Mirror } \\
\hline Major Axis: & $55.8 \mathrm{~cm}$ \\
\hline Minor Axis: & $2.2 \mathrm{~cm}$ \\
\hline Incoming angle: & $4^{\circ}$ \\
\hline \multicolumn{2}{|c|}{$\begin{array}{l}\text { Illumination Distance: } 3 \mathrm{~cm} \\
\text { (RIXS) }\end{array}$} \\
\hline $\begin{array}{l}\text { Illumination Dist } \\
\text { (Time-resolved XES) }\end{array}$ & $: 1.8 \mathrm{~cm}$ \\
\hline
\end{tabular}

Figure 10: Schematic of the proposed design, including summary of the component parameters. 


\section{TABLES}

Table 1: The maximum source angular divergence that allows $200 \mu \mathrm{m}$ source resolution for a $5 \mu \mathrm{m}$ source offset $500 \mu \mathrm{m}$ at different magnifications of the source. From this data we determine the best position of the non-dispersive focusing mirror to be that which allows 1:10 imaging.

\begin{tabular}{ccc}
\hline \hline Magnification & Sample Resolution & Source Divergence (rad) \\
\hline $1: 10$ & $2 \mu \mathrm{mm}$ & 0.011 \\
$1: 7$ & $1.4 \mathrm{~mm}$ & 0.011 \\
$1: 5$ & $1 \mathrm{~mm}$ & 0.011 \\
$1: 3$ & $600 \mu \mathrm{m}$ & 0.011 \\
$1: 2$ & $400 \mu \mathrm{m}$ & 0.009 \\
$1: 1$ & $200 \mu \mathrm{m}$ & 0.007 \\
$2: 1$ & $100 \mu \mathrm{m}$ & 0.005 \\
$3: 1$ & $66 \mu \mathrm{m}$ & 0.0035 \\
$5: 1$ & $40 \mu \mathrm{m}$ & 0.0023 \\
$7: 1$ & $29 \mu \mathrm{m}$ & 0.0017 \\
$10: 1$ & $20 \mu \mathrm{m}$ & 0.0013 \\
\hline
\end{tabular}


Table 2: The results of simulations in which different grating and mirror illumination lengths were tested to determine which combinations produce the necessary resolution for the instrument. In the time-resolved XES case, we used a point source offset $50 \mu \mathrm{m}$ from the center to simulate a $100 \mu \mathrm{m}$ source; for the RIXS the offset was $500 \mu \mathrm{m}$ for a $1 \mathrm{~mm}$ source. Blue cells represent those combinations which yield an acceptable resolution. Yellow cells appear to have acceptable resolution but have grating illuminations at which there is significant image error.

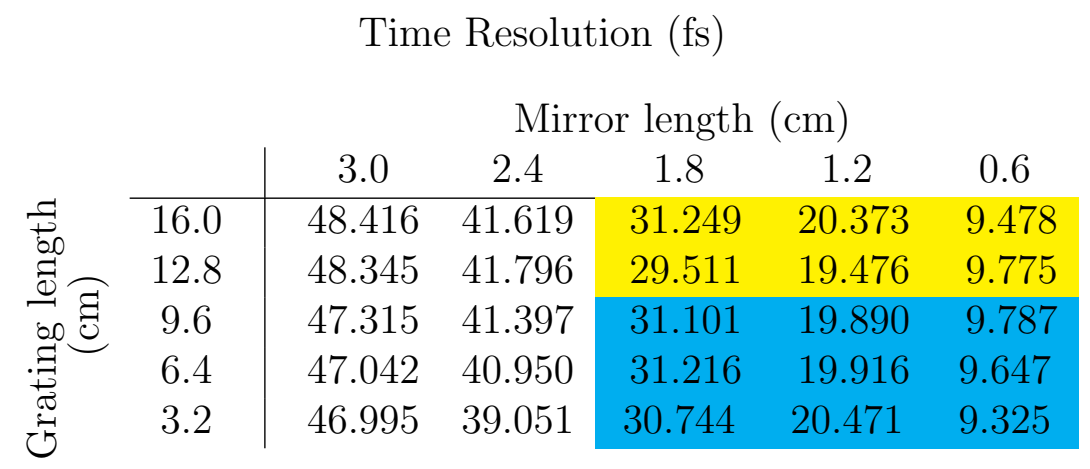

\begin{tabular}{|c|c|c|c|c|c|}
\hline \multicolumn{6}{|c|}{ Energy Resolution (eV) } \\
\hline & \multicolumn{5}{|c|}{ Mirror length $(\mathrm{cm})$} \\
\hline & 3.0 & 2.4 & 1.8 & 1.2 & 0.6 \\
\hline 16.0 & 0.1230 & 0.1041 & 0.0859 & 0.0678 & 0.0335 \\
\hline 12.8 & 0.1230 & 0.1038 & 0.0857 & 0.0674 & 0.0333 \\
\hline 9.6 & 0.1231 & 0.1032 & 0.0860 & 0.0680 & 0.0340 \\
\hline 6.4 & 0.1227 & 0.1034 & 0.0860 & 0.0673 & 0.0333 \\
\hline 3.2 & 0.1215 & 0.1034 & 0.0839 & 0.0667 & 0.0333 \\
\hline
\end{tabular}

